\title{
Ground-penetrating-radar response to fracture-fluid salinity: Why lower frequencies are favorable for resolving salinity changes
}

\author{
Georgios P. Tsoflias ${ }^{1}$ and Matthew W. Becker ${ }^{2}$
}

\begin{abstract}
Time-lapse ground-penetrating-radar (GPR) surveys exploit signal-amplitude changes to monitor saline tracers in fractures and to identify groundwater flow paths. However, the relationships between GPR signal amplitude, phase, and frequency with fracture aperture and fluid electrical conductivity are not well understood. We used analytical modeling, numerical simulations, and field experiments of multifrequency GPR to investigate these relationships for a millimeter-scale-aperture fracture saturated with water of varying salinity. We found that the response of lower-frequency radar signals detects changes in fluid salinity better than the response of higher-frequency signals. Increasing fluid electrical conductivity decreases low-frequency GPR signal wavelength, which improves its thin-layer resolution capability. We concluded that lower signal frequencies, such as $50 \mathrm{MHz}$, and saline tracers of up to $1 \mathrm{~S} / \mathrm{m}$ conductivity are preferable when using GPR to monitor flow in fractured rock. Furthermore, we found that GPR amplitude and phase responses are detectable in the field and predictable by EM theory and modeling; therefore, they can be related to fracture aperture and fluid salinity for hydrologic investigations of fractured-rock flow and transport properties.
\end{abstract}

\section{INTRODUCTION}

Predicting flow and transport in fractured bedrock remains a challenge (National Research Council, 1996). Flow through this highly heterogeneous medium is controlled by fracture aperture, channeling along the fracture (i.e., aperture variability), and fracture connectivity. Ground-penetrating radar (GPR) is a suitable near-surface geophysical method for detecting and imaging fractures (Davis and Annan, 1989; Grasmueck, 1996; Seol et al., 2001; Tsoflias et al., 2004; Jeannin et al., 2006). Surface reflection and borehole tomogra- phy studies relate GPR signal amplitude to fracture aperture and fluid content (Lane et al., 1998; Lane et al., 2000; Grégoire et al., 2003; Grégoire and Hollender, 2004). Time-lapse GPR methods have successfully monitored fractured aquifer hydraulic testing (Tsoflias et al., 2001) and flow paths by imaging saline tracers introduced to discrete fractures (Day-Lewis et al., 2003; Talley et al., 2005). These investigations detect increased GPR reflection amplitude when saline water is injected in a freshwater-filled fracture and monitor salinefluid flow based on relative GPR amplitude changes. In porous media, Hagrey and Muller (2000) show an increase in GPR reflected amplitudes correlating to increased pore-fluid salinity.

Although investigators have detected increased GPR amplitude related to increased fluid salinity, the relationships between GPR signal amplitude, phase, and frequency with fracture aperture and fluid salinity have not been investigated adequately nor related quantitatively. Furthermore, most of the literature has investigated the amplitude response of GPR signals, offering limited insight in the utility of signal phase to fracture and fluid characterization. Better understanding of radar amplitude and phase response to fluid salinity can result in (a) improved geophysical determination of hydrologically important parameters such as fracture aperture and tracer concentration and (b) appropriate selection of GPR survey parameters for monitoring saline tracers in fractured formations.

Our objective is to investigate the response of multifrequency GPR signal amplitude and phase to millimeter-scale fracture apertures saturated with water of varying salinity. We do this by analytical and numerical modeling and by field experiments. We model fractures as thin, parallel plate layers. In our field experiments, we exploit an extensively studied fractured-bedrock site with hydraulically conductive subhorizontal fractures. We find that lower-frequency GPR signals exhibit greater changes in response to water electrical conductivity changes in a discrete fracture.

\section{BACKGROUND}

Electromagnetic (EM) wave propagation is discussed in numerous texts (Stratton, 1941; Balanis, 1989). In layered media, radar-

Manuscript received by the Editor 7 January 2008; revised manuscript received 5 April 2008; published online 26 August 2008.

${ }^{1}$ The University of Kansas, Lawrence, Kansas, U.S.A. E-mail: tsoflias@ku.edu.

${ }^{2}$ University at Buffalo, State University of New York, Buffalo, New York, U.S.A. E-mail: mwbecker@ geology.buffalo.edu. (C) 2008 Society of Exploration Geophysicists. All rights reserved. 
wave reflection and transmission is a function of permittivity $\varepsilon$, electrical conductivity $\sigma$, magnetic permeability $\mu$, and layer thickness as well as EM wavefield frequency $f$, angle of incidence, and polarization. Radar reflections originate at dielectric boundaries in the subsurface that commonly coincide with layered-earth interfaces. The amplitude and phase of reflected radar signals depend on the parameters listed above and are described by the Fresnel equations (Stratton, 1941; Balanis, 1989). In GPR studies, the amplitude response of thin layers has been examined in terms of layer thickness, EM wave angle of incidence, and polarization (Arcone et al., 2004; Grégoire and Hollender, 2004; Bradford and Deeds, 2006). In addition to the GPR amplitude response to these parameters, Hollender and Tillard (1998) and Tsoflias and Hoch (2006) consider multiple frequencies and examine the phase response of GPR signals to thin layers.

Of interest to our work is the EM response to a thin layer with thickness orders-of-magnitude smaller than signal wavelength (millimeter-scale fractures of varying aperture imaged by decimeter-to meter-scale signal wavelengths) and filled with water of varying electrical conductivity. We consider a nonmagnetic environment with negligible polarization effects from the homogeneity of the rock matrix and the small angle of EM wave incidence to the horizontal fracture plane $\left(<10^{\circ}\right)$ at a field site. The effect of thin-layer thickness to normally incident waves is well understood as an interference phenomenon between reflected waves from the upper and lower interfaces (Widess, 1973). For a fracture enclosed in a homogeneous matrix, reflection amplitude is expected to increase from a value of zero for zero layer thickness to maximum reflection strength when layer thickness is approximately equal (depending on wavelet
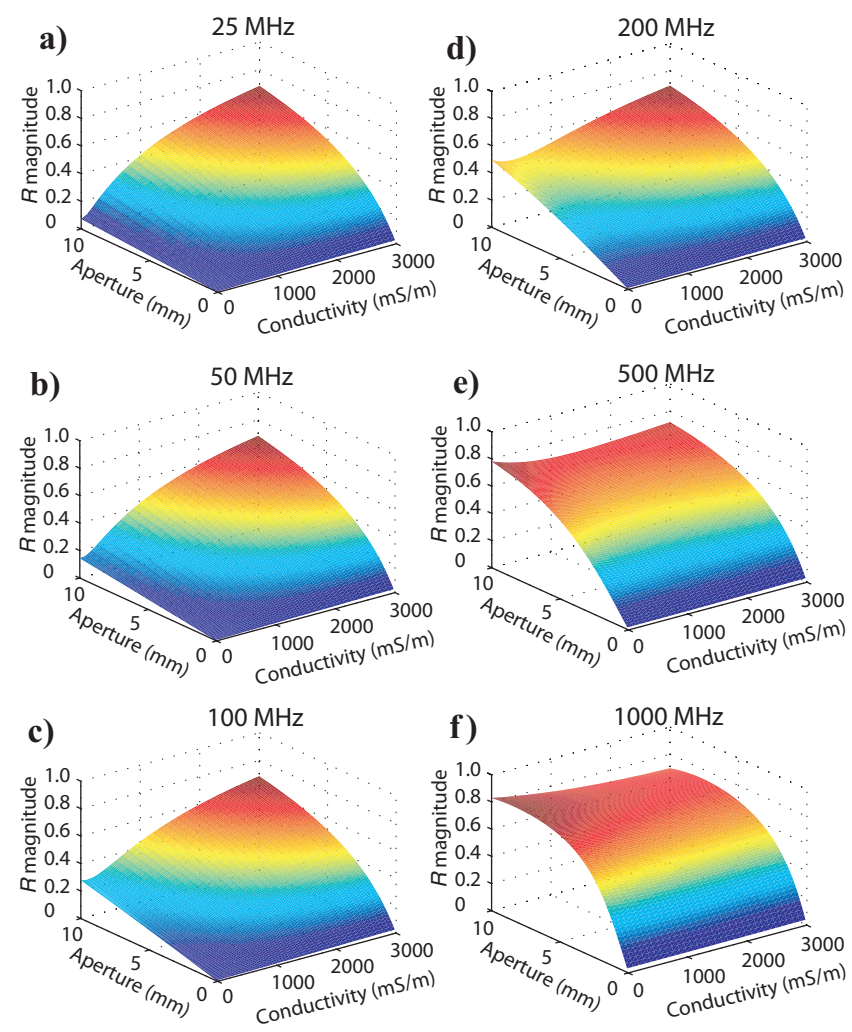

Figure 1. Plots of reflection coefficient magnitude $R$ as a function of fracture aperture and water electrical conductivity for frequencies of (a) 25, (b) 50, (c) 100, (d) 200, (e) 500, and (f) $1000 \mathrm{MHz}$. shape) to one-fourth of signal wavelength $\lambda$ in the medium filling the fracture. Higher-frequency signals have shorter wavelengths and can resolve thinner layers. Therefore, in our study of millimeterscale fractures, higher GPR frequencies are expected to image fracture properties better than lower frequencies.

In addition to the well-known effect of layer thickness on reflection amplitude, EM theory predicts that electrically conductive (lossy) media yield complex reflection and transmission coefficients that imply a phase shift (Stratton, 1941). The Fresnel equations describe the dependence of the complex reflection coefficient on layer thickness, electrical conductivity, permittivity, and signal frequency. At a fractured geologic setting, we expect that a saline tracer will cause a characteristic change to the reflected GPR signal amplitude and phase. Furthermore, the characteristics of the reflection will depend on the frequency of the signal.

\section{METHODS AND RESULTS}

We use analytical modeling, numerical simulations, and tracer experiments at a fractured sandstone field site to examine the relationships of multifrequency GPR amplitude and phase response to millimeter-scale fractures saturated with saline water.

\section{Analytical modeling}

\section{Methods}

We employed the recursive method used in EM plane-wave studies that considers field continuity and matches the impedances across boundaries to analytically compute the reflection-coefficient magnitude and phase responses of layered media (Balanis, 1989). Model parameters were selected to match field conditions. We considered varying fracture apertures $(0-10 \mathrm{~mm})$ and water salinity $(0-3 \mathrm{~S} / \mathrm{m})$ for a range of frequencies $(25,50,100,200,500$, and $1000 \mathrm{MHz}$ ) commonly used in GPR studies. Fractured-rock-matrix relative permittivity and electrical conductivity were set to 7 and $0.1 \mathrm{mS} / \mathrm{m}$, respectively; water relative permittivity to 80 ; and EM wave angle of incidence to $7.5^{\circ}$ to simulate field acquisition geometry. Because of low angle of incidence, wavefield polarization has negligible effects on the computed reflection coefficients; therefore, only transverse electric (TE) polarization results are presented. We assumed that medium constitutive parameters are independent of frequency in the GPR frequency range (Davis and Annan, 1989).

\section{Results}

Figure 1 plots reflection coefficient magnitude $R$ as a function of fracture aperture $\delta$ and water electrical conductivity $\sigma$ for a range of frequencies $f$.

Dependence of $R$ on fracture aperture. - For all conductivities and frequencies, increasing fracture aperture in turn increases the magnitude of $R$. For low $\sigma(<1 \mathrm{~S} / \mathrm{m})$, higher frequencies result in greater changes in $R$ as a function of $\delta$, i.e., higher frequencies offer greater response to layer-thickness change than lower frequencies. However, for high $\sigma, R$ displays a comparable response as a function 
of $\delta$ at all frequencies. Thus, at higher $\sigma$, the relationship between $R$ and fracture aperture is relatively invariable with frequency. This observation is counterintuitive because in wave phenomena we expect higher frequencies to offer higher resolution.

Dependence of $R$ on electrical conductivity. - For low and intermediate frequencies $(25-200 \mathrm{MHz})$, increasing $\sigma$ results in greater change in $R$ compared with high frequencies (500 and $1000 \mathrm{MHz}$ ). Higher frequencies exhibit lower dependence of $R$ to change in water electrical conductivity for all apertures. Therefore, lower frequencies offer a better ability to detect GPR amplitude changes related to fluid electrical conductivity changes.

Figure 2 illustrates reflection coefficient phase $\phi$ as a function of $\delta$ and $\sigma$ for a range of $f$.

Dependence of $\phi$ on fracture aperture. - For low frequencies, there is no significant change of $\phi$ as a function of aperture, whereas high frequencies show $\phi$ change of up to $-90^{\circ}$ (phase lag) as layer thickness approaches one-quarter wavelength.

Dependence of $\phi$ on electrical conductivity. - For all frequencies, increased fluid electrical conductivity results in increased phase delay. Low frequencies show rapid phase change when $\sigma$ increases from 0 to $1 \mathrm{~S} / \mathrm{m}$, whereas intermediate frequencies show similar rapid change of phase when $\sigma$ increases up to $2 \mathrm{~S} / \mathrm{m}$. High frequencies show less $\phi$ dependence to $\sigma$. Therefore, lower and intermediate frequencies exhibit a better ability to detect changes in GPR phase related to changes in fluid electrical conductivity.

A direct comparison of these relationships is shown in Figure 3, which plots multifrequency amplitude and phase response as a function of aperture for low $(100 \mathrm{mS} / \mathrm{m})$ and high $(2500 \mathrm{mS} / \mathrm{m})$ water electrical conductivities (Figure $3 \mathrm{a}, \mathrm{b}, \mathrm{d}$, and e) and as a function of conductivity for a 1-mm aperture (Figure $3 \mathrm{c}$ and f). Figure $3 \mathrm{~b}$ shows 25-, 50-, 100-, and 200 -MHz frequencies exhibiting nearly identical $R$ for millimeter-scale fractures saturated with $2.5 \mathrm{~S} / \mathrm{m}$ water. The reduced change of amplitude and phase of the high-frequency signals (500 and $1000 \mathrm{MHz}$ ) as a function of electrical conductivity is seen in Figure $3 \mathrm{c}$ and $\mathrm{f}$.

Additional insight about the effect of thin-layer electrical conductivity on the response of EM waves is gained by examining changes in $\lambda$. The wavelength of layer $n$ of the subsurface is computed by

$$
\lambda_{n}=\frac{2 \pi}{k_{n}},
$$

where $k_{n}$ is the complex wavenumber

$$
k_{n}=\sqrt{\omega^{2} \mu_{n} \varepsilon_{n}-j \omega \mu_{n} \sigma_{n}}
$$

and $\omega$ is the angular frequency. Figure 4 plots wavelength as a function of water electrical conductivity for 25-1000-MHz-frequency signals. At low frequencies, signal wavelength decreases rapidly; it approaches that of higher frequencies as conductivity increases. High frequencies show
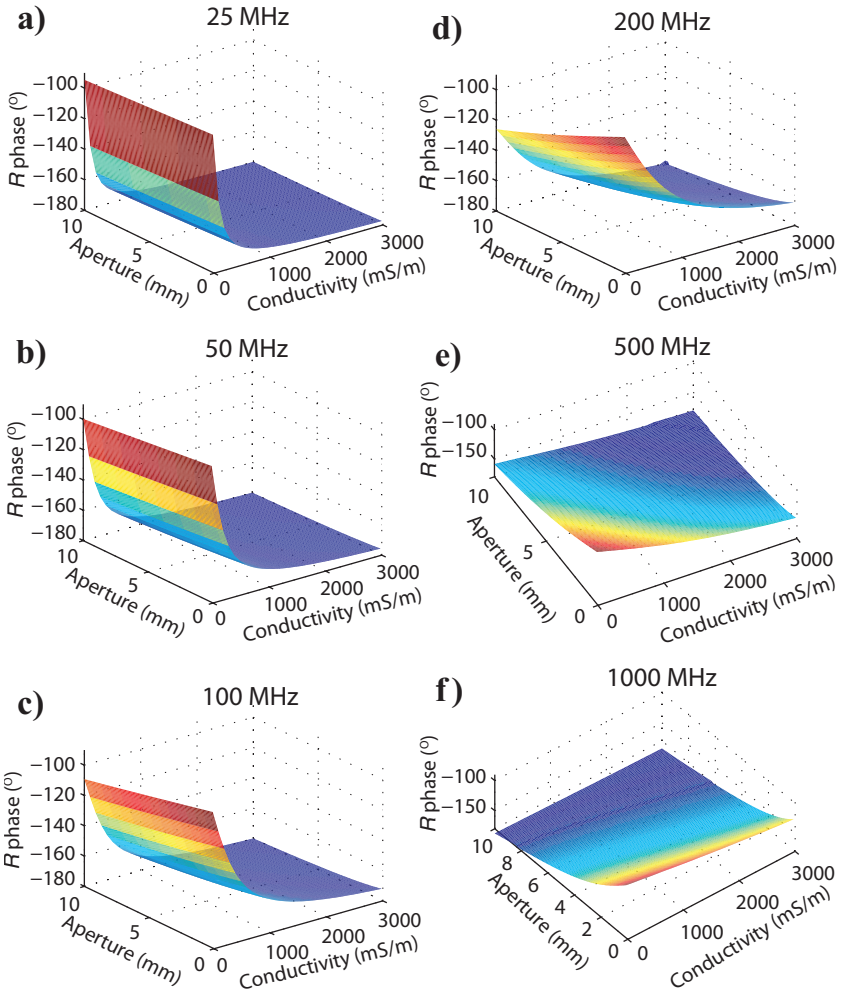

Figure 2. Plots of reflection coefficient phase $\phi$ as a function of fracture aperture and water electrical conductivity for frequencies of (a) 25, (b) 50, (c) 100, (d) 200, (e) 500, and (f) $1000 \mathrm{MHz}$ a)

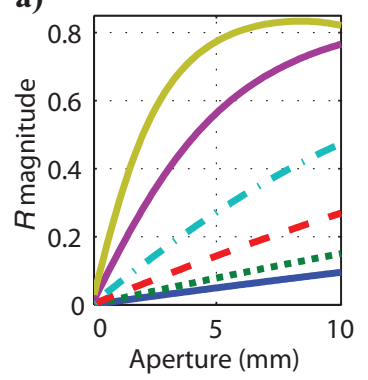

d)

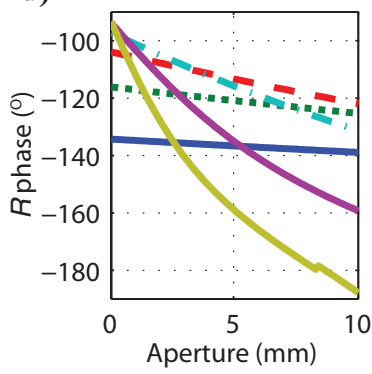

e)
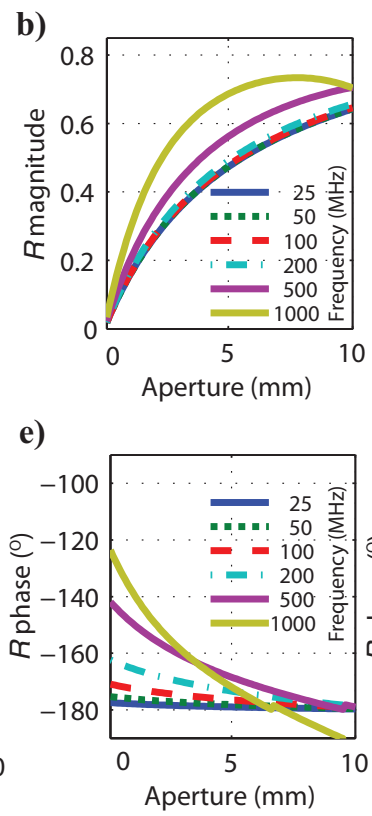

Aperture $1 \mathrm{~mm}$

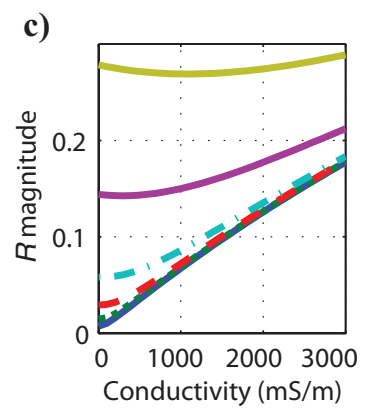

f)

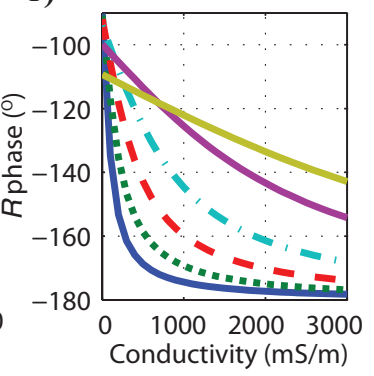

Figure 3. Multifrequency amplitude and phase response of reflection coefficient as a function of fracture aperture for low $(100 \mathrm{mS} / \mathrm{m})$ and high $(2500 \mathrm{mS} / \mathrm{m})$ water electrical conductivities $(\mathrm{a}, \mathrm{b}, \mathrm{d}, \mathrm{e})$, and as a function of conductivity for 1-mm aperture (c, f). 
very small $\lambda$ change (decrease) as a function of increase in $\sigma$. This wavelength response results in the greater sensitivity of $R$ to conductivity at lower frequencies than higher frequencies (Figures 1 and $3 b)$.

\section{Numerical modeling}

\section{Methods}

To relate field results to theoretical expectations, we used GprMax2D (Giannopoulos, 2002), a GPR simulator based on the finite-difference time-domain (FDTD) method. Numerical simulations incorporate propagation effects, such as signal attenuation and dispersion, not captured by analytical reflection-coefficient modeling. Modeling parameters were set to represent a fractured sandstone environment similar to the field site investigated. Rock-matrix parameters were $\varepsilon_{r}=7, \sigma=0.1 \mathrm{mS} / \mathrm{m}$, and water $\varepsilon_{r}=80$. Water electrical conductivities were set to low $(28 \mathrm{mS} / \mathrm{m})$, intermediate $(400 \mathrm{mS} / \mathrm{m})$, and high $(2500 \mathrm{mS} / \mathrm{m})$, and source signal frequencies were set to 50 and $100 \mathrm{MHz}$ as in the experimental field setup. Modeled fracture apertures were $0.5,1,2$, and $10 \mathrm{~mm}$. Model grid cell size was $0.5 \mathrm{~mm}$ in two dimensions, and the source pulse was simulated by a Ricker wavelet.

\section{Results}

Numerical modeling results exhibit the same responses predicted by the analytical models and are in general agreement with numeri-

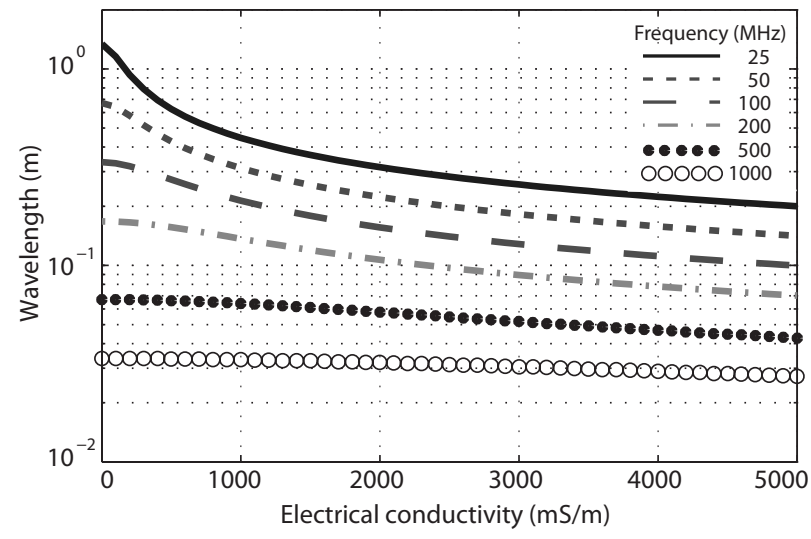

Figure 4. Wavelength as a function of water electrical conductivity for 25-, 50-, 100-, 200-, 500-, and 1000-MHz frequency signals.
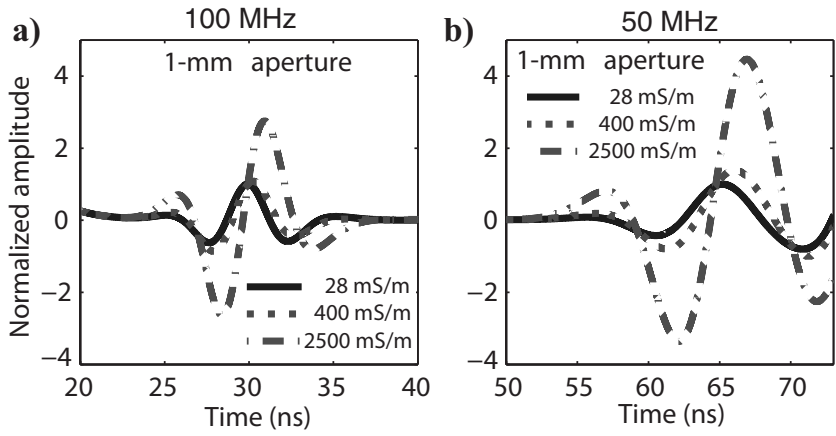

Figure 5. Numerically simulated (a) 100- and (b) 50-MHz GPR traces reflected from a 1-mm-aperture fracture, saturated with low-, medium-, and high-salinity water of 28,400 , and $2500 \mathrm{mS} / \mathrm{m}$, respectively. cal GPR models in fractured granite presented by Lane et al. (2000). Overall, reflection amplitudes increase with increasing frequency, aperture, and fluid electrical conductivity. Phase lag increases with increasing water salinity.

Of interest to this study is a comparison of amplitude and phase changes between varying frequency data as a function of fracturewater salinity. Normalizing the reflections within each frequency to the reflection observed at background conductivity allows us to compare relative amplitude changes between the $50-$ and $100-\mathrm{MHz}$ frequency data.

Figure 5 displays 50- and 100-MHz waveforms, each normalized to the maximum amplitude at background salinity $(28 \mathrm{mS} / \mathrm{m})$. Both frequency sets of waveforms show that increased water salinity in turn increases reflection amplitude and phase lag (apparent time delay). However, the $50-\mathrm{MHz}$ signals display greater amplitude change from background than the $100-\mathrm{MHz}$ signals. Therefore, in a tracer-monitoring investigation, lower frequencies would offer a greater ability to detect salinity changes based on signal amplitude changes. Phase delay would also exhibit strong dependence on water electrical conductivity and signal frequency.

\section{Field experiments}

\section{Methods}

Variable salinity tracers injected into a subhorizontal fracture were monitored using multifrequency GPR reflection at the Altona Flat Rock site, $15 \mathrm{~km}$ northwest of Plattsburgh, New York. The field site has been equipped for fractured-rock hydrologic studies and was used for time-lapse GPR monitoring of saline tracers by Talley et al. (2005). The site is underlain by the Cambrian Potsdam Sandstone, a highly lithified quartzose sandstone with laterally extensive subhorizontal bedding-plane partitions. Soil overburden is thin or nonexistent at the site because of a glacial dam burst at the end of the last Pleistocene glacial retreat (Rayburn et al., 2005).

A hydraulically conductive subhorizontal fracture $7.6 \mathrm{~m}$ below ground surface with a mean hydraulic aperture of $0.5 \mathrm{~mm}$ (Talley et al., 2005) was investigated in this experiment. To monitor the effect of varying water electrical conductivity to radar response as a function of frequency, surface reflection GPR data were acquired using two (50- and 100-MHz) PulseEKKO100 stationary GPR systems. The antennas were positioned over the same midpoint location between an injection well (404) and a pumping well (304) drilled $7 \mathrm{~m}$ apart, parallel to the general direction of groundwater flow. Inflatable PVC packers were used to isolate the fracture hydraulically.

Adipole flow pattern was induced by pumping well 304 at a rate of approximately 5 liters per minute and reinjecting the extracted water in well 404 via a 20-liter reservoir at the surface. A saline tracer was introduced to the system by adding table salt $(\mathrm{NaCl})$ to the surface reservoir. An increasing amount of $\mathrm{NaCl}$ was added to the flow system incrementally, and the electrical conductivity of the pumped water was monitored in the surface reservoir to ensure complete mixing of the tracer in the fracture and surface reservoir. Fracturewater electrical conductivity equilibrated at 138, 312, 620, 755, 1200 , and $1640 \mathrm{mS} / \mathrm{m}$ during the sequential tracer injections. Fifty radar traces were recorded simultaneously with each GPR system during each of the fracture-water conductivity levels. 


\section{Results}

Figure 6 displays 100- and 50-MHz GPR traces acquired during the tracer-injection experiment. Color-coded traces correspond to different water electrical conductivity levels. A gain factor of [time $]^{2.5}$ is applied to the data for display purposes, and low-pass Butterworth filtering is used to remove high-frequency noise. The reflected waveform from the 7.6-m water-saturated fracture is at approximately 140-ns two-way traveltime (identified by arrows in Figure 6). The 50-MHz data show increasing signal amplitude and increasing phase lag (apparent time delay), corresponding to increasing fluid conductivity. The signal changes are evident through the entire waveform (peak-trough-peak) of the fracture reflection. Radar traces show good repeatability of waveforms before the fracture reflection, suggesting that changes observed at the level of the fracture result from changes in water electrical conductivity. The 100 -MHz data display distinct amplitude and phase responses that are analogous to the 50-MHz data but of lesser magnitude.

Extracting the maximum amplitude of the 140-ns fracture reflector from each GPR trace during the tracer experiment indicates the 50-MHz frequency data exhibit greater amplitude changes as a function of water electrical conductivity change compared with the 100$\mathrm{MHz}$ data (Figure 7). Figure 7a plots reflector amplitudes normalized to (i.e., divided by) the mean amplitude of traces corresponding to $138 \mathrm{mS} / \mathrm{m}$ conductivity (referred to as background) for each frequency. Normalizing each frequency to a corresponding background reflection removes amplitude differences between the 100- and 50 -MHz data sets associated with instrumentation, power output, and frequency-dependent propagation effects (attenuation and dispersion). Figure $7 \mathrm{~b}$ plots rescaled reflector amplitudes with the average amplitude of background conductivity traces subtracted from each trace. Figure $7 \mathrm{~b}$ shows even greater amplitude difference between 50- and $10-\mathrm{MHz}$ recordings, the result of lower-frequency systems transmitting more power into the subsurface and suffering less attenuation than higher frequencies. A direct consequence is greater sig-

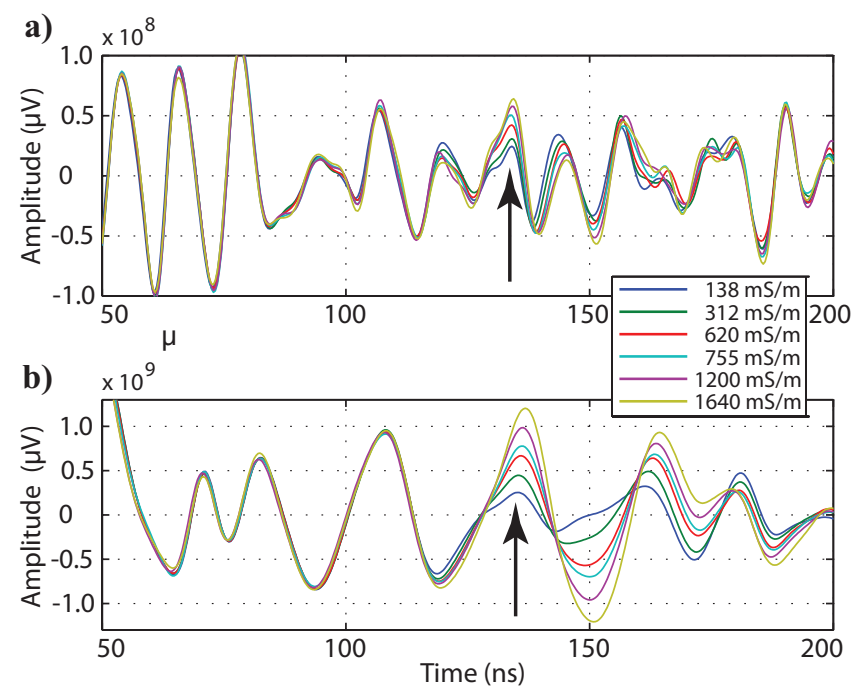

Figure 6. Depiction of (a) 100- and (b) 50-MHz GPR traces corresponding to varying fracture-water electrical conductivity: $138 \mathrm{mS} / \mathrm{m}$, trace $25 ; 312 \mathrm{mS} / \mathrm{m}$, trace $75 ; 620 \mathrm{mS} / \mathrm{m}$, trace 125 ; $755 \mathrm{mS} / \mathrm{m}$, trace $175 ; 1200 \mathrm{mS} / \mathrm{m}$, trace $225 ; 1640 \mathrm{mS} / \mathrm{m}$. trace 275. nal-to-noise ratio content in the 50-MHz frequency data than in the 100-MHz data (Figure 7a).

The phase response of the fracture reflection to varying tracer concentration is assessed by evaluating the complex trace phase angles of the time series (Taner et al., 1979). Figure $8 \mathrm{a}$ and b shows the complex trace phase difference between each of the traces displayed in Figure 6 and background trace $25(138 \mathrm{mS} / \mathrm{m})$. Increasing phase lag corresponds to increasing water electrical conductivity at both 50and $100-\mathrm{MHz}$ frequencies. Figure $8 \mathrm{c}$ plots the complex trace phase difference between each reflection during the tracer test and the average complex trace phase corresponding to $138 \mathrm{mS} / \mathrm{m}$. Overall, greater signal phase delay as a function of water electrical conductivity is exhibited by the $50-\mathrm{MHz}$ data compared with the $100-\mathrm{MHz}$ data. The $50-\mathrm{MHz}$ signal phase change is greater at smaller conductivity ranges, and it decreases in magnitude with increasing electrical conductivity. The $100-\mathrm{MHz}$ signal exhibits relatively uniform phase change across the range of conductivities considered.

Note that Figures $7 \mathrm{a}$ and $8 \mathrm{c}$ reveal small trends of amplitude and phase change during each of the incremental tracer tests, which re- a)

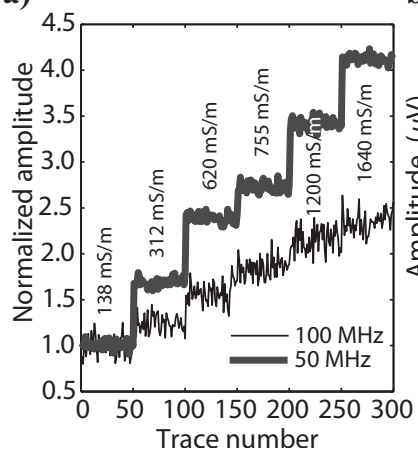

b)

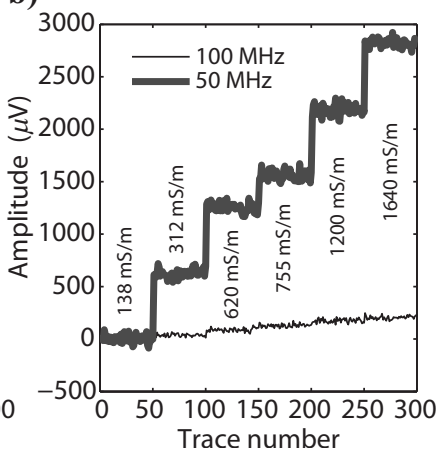

Figure 7. Fracture reflector amplitude (a) normalized to mean background and (b) rescaled to mean background. Water electrical conductivities are marked over corresponding traces: $138 \mathrm{mS} / \mathrm{m}$, traces $1-50 ; 312 \mathrm{mS} / \mathrm{m}$, traces $51-100 ; 620 \mathrm{mS} / \mathrm{m}$, traces $101-150 ; 755$ $\mathrm{mS} / \mathrm{m}$, traces $151-200 ; 1200 \mathrm{mS} / \mathrm{m}$, traces $201-250 ; 1640 \mathrm{mS} / \mathrm{m}$, traces 251-300.
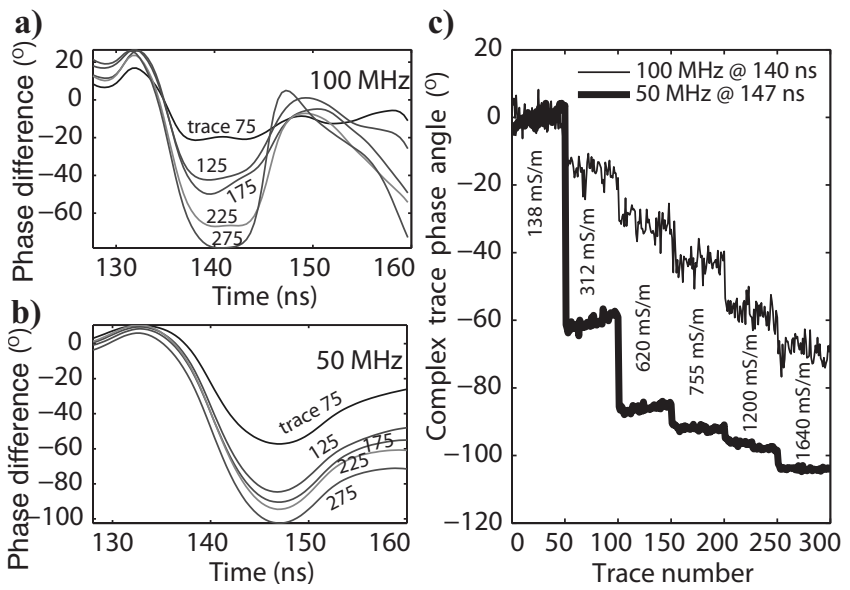

Figure 8. Complex trace phase angle difference between trace 25 and traces $75,125,175,225$, and 275 at (a) $100-$ and (b) $50-\mathrm{MHz}$ frequencies. (c) Complex trace phase difference from mean background corresponding to $138 \mathrm{mS} / \mathrm{m}$. 
sult from small tracer-concentration changes. For example, during the $620-\mathrm{mS} / \mathrm{m}$ tracer test, starting water electrical conductivity was $634 \mathrm{mS} / \mathrm{m}$ and ending conductivity was $605 \mathrm{mS} / \mathrm{m}$. The 29-mS/m conductivity decline between traces 101 and 150 results in the trends of decreasing amplitude and increasing phase.

\section{CONCLUSIONS}

Multifrequency EM analytical modeling, numerical simulations, and GPR field experiments show good agreement relating the response of GPR signals to fracture-fluid salinity. We cite three findings. First, increasing fluid electrical conductivity causes lower-frequency signals to exhibit greater amplitude and phase-delay changes than higher frequencies. Second, the phase response of GPR to saline fluids is as characteristic as the amplitude response. Finally, higher energy output and lower attenuation of low-frequency systems $(50 \mathrm{MHz})$ yield greater amplitude reflections from fractures saturated with electrically conductive fluids and greater signal-tonoise compared with high-frequency systems (100 MHz).

These findings suggest that lower-frequency GPR should be used to monitor saline-tracer flow in discrete fractures. This finding contradicts accepted wisdom that thin-layer properties are better resolved by high-frequency signals. In addition, these findings suggest that both phase and amplitude should be used to image bedrock fractures. Currently, signal phase is neglected in field studies.

Our investigation shows that characteristic and quantifiable GPR amplitude and phase responses can be related to fracture properties. Trends and magnitudes of modeled amplitude and phase changes (Figure $3 \mathrm{c}$ and $\mathrm{f}$ and Figure 5) compared favorably with field observations (Figures $7 \mathrm{a}$ and $8 \mathrm{c}$ ) despite uncertainty in the actual (mechanical) fracture aperture. This suggests that amplitude and phase data may be used to decouple measurements of aperture and conductivity where both vary in fractures. However, it is important to note that bedrock fracture apertures can vary by orders of magnitude at the decimeter scale. Thus, the aperture detected by radar represents a spatial average as sampled by the propagating waveform, and Fresnel zone effects can be significant. This can lead to an artificial dilution of saline concentration as measured using GPR. Therefore, mapping heterogeneous transport of saline fluid through a fracture requires careful consideration of GPR spatial resolution.

Inversion of time-lapse, multifrequency GPR amplitude and phase data holds promise for uniquely determining fracture aperture distribution and tracer concentration of a discrete fracture plane. Our finding that fracture imaging is enhanced by using low-frequency radar suggests that it may be possible to image bedrock fractures in deeper environments than have been considered. In addition, it suggests that borehole radar imaging of bedrock fractures may also benefit from lower-frequency antennas and careful consideration of signal phase and amplitude.

\section{ACKNOWLEDGMENTS}

We thank students Greg Babonis, Shannon Renee Bourque, Jeremy Crowley, and Karina Kervin for assistance in this study. We are grateful to Greg Baker, Dave Franzi, and the Miner Institute for access to the field site and their continued support and guidance. Funding for this research was provided by the American Chemical Society PRF 43401-G8 and the National Science Foundation grant GEO-
0207720. Acquisition of the GPR system was funded by the National Science Foundation (EAR/IF-0345445). The Altona site was established with support from the National Science Foundation (EAR0207720).

\section{REFERENCES}

Arcone, S. A., V. B. Spikes, G. S. Hamilton, and P. A. Mayewski, 2004, Stratigraphic continuity in $400 \mathrm{MHz}$ short-pulse radar profiles of firn in west Antarctica: Annals of Glaciology, 39, 195-200, doi: 10.3189/ 172756404781813925.

Balanis, C., 1989, Advanced engineering electromagnetics: John Wiley \& Sons, Inc.

Bradford, J. H., and J. C. Deeds, 2006, Ground penetrating radar theory and application to thin-bed offset-dependent reflectivity: Geophysics, 71, no. 3, K47-57.

Davis, J. L., and A. P. Annan, 1989, Ground penetrating radar for high-resolution mapping of soil and rock stratigraphy: Geophysical Prospecting, 37, 531-551

Day-Lewis, F. D., J. W. Lane Jr., J. M. Harris, and S. M. Gorelick, 2003, Time-lapse imaging of saline-tracer transport in fractured rock using difference-attenuation radar tomography: Water Resources Research, 39, 1290.

Giannopoulos, A., 2002, GPRMAX2D/3D user's manual, v. 1.5, http://www.gprmax.org, accessed June 2008.

Grasmueck, M., 1996, 3-D ground-penetrating radar applied to fracture imaging in gneiss: Geophysics, 61, 1050-1064.

Grégoire, C., L. Halleux, and V. Lukas, 2003, GPR abilities for the detection and characterization of open fractures in a salt mine: Near Surface Geophysics, 1, 139-147.

Grégoire, C., and F. Hollender, 2004, Discontinuity characterization by the inversion of the spectral content of ground-penetrating-radar (GPR) reflection: Application of the Jonscher model: Geophysics, 69, 1414-1424.

Hagrey, S. A., and C. Muller, 2000, GPR study of pore water content and salinity in sand: Geophysical Prospecting, 48, 63-85.

Hollender, F., and S. Tillard, 1998, Modeling ground-penetrating radar wave propagation and reflection with the Jonscher parameterization: Geophysics, 63, 1933-1942.

Jeannin, M., S. Garambois, C. Grégoire, and D. Jongmans, 2006, Multiconfiguration GPR measurements for geometric fracture characterization in limestone cliffs (Alps): Geophysics, 71, no. 5, B85-B92.

Lane, J. W. Jr., M. L. Buursing, F. P. Haeni, and R. J. Versteeg, 2000, Evaluation of ground-penetrating radar to detect free-phase hydrocarbons in fractured rocks - Results of numerical modeling and physical experiments: Ground Water, 38, 929-938.

Lane, J. W. Jr., F. P. Haeni, and F. D. Day-Lewis, 1998, Use of time-lapse attenuation-difference radar tomography methods to monitor saline tracer transport in fractured crystalline bedrock: Proceedings of the 7th International Conference on Ground Penetrating Radar, 2, 533-538.

National Research Council, 1996, Rock fractures and fluid flow: Contemporary understanding and applications: National Academy Press.

Rayburn, J. A., P. L. K. Knuepfer, and D. A. Franzi, 2005, A series of large, late Wisconsinan meltwater floods through the Champlain and Hudson Valleys, New York State, USA: Quaternary Science Reviews, 24, 2410-2419.

Seol, S. J., J.-H. Kim, Y. Song, and S.-H. Chung, 2001, Finding the strike direction of fractures using GPR: Geophysical Prospecting, 49, 300-308.

Stratton, J. A., 1941, Electromagnetic theory: McGraw-Hill Book Company.

Talley, J., G. S. Baker, M. W. Becker, and N. Beyrle, 2005, Four dimensional mapping of tracer channelization in subhorizontal bedrock fractures using surface ground penetrating radar: Geophysics Research Letters, 32, L04401.

Taner, M. T., F. Koehler, and R. E. Sheriff, 1979, Complex seismic trace analysis: Geophysics, 44, 1041-1063.

Tsoflias, G. P., T. Halihan, and J. M. Sharp Jr., 2001, Monitoring pumping test response in a fractured aquifer using ground penetrating radar: Water $\mathrm{Re}$ sources Research, 37, 1221-1229.

Tsoflias, G. P., and A. Hoch, 2006, Investigating multi-polarization GPR wave transmission through thin layers: Implications for vertical fracture characterization: Geophysics Research Letters, 33, L20401.

Tsoflias, G. P., J. P. Van Gestel, P. Stoffa, D. Blankenship, and M. Sen, 2004, Vertical fracture detection by exploiting the polarization properties of GPR signal: Geophysics, 69, 803-810.

Widess, M. B., 1973, How thin is a thin bed: Geophysics, 38, 1176-1180. 